Relations industrielles

Industrial Relations

\title{
Propos du lecteur
}

\section{L'avenir de la Loi de la convention collective}

Volume 4, numéro 6, février 1949

URI : https://id.erudit.org/iderudit/1023494ar

DOI : https://doi.org/10.7202/1023494ar

Aller au sommaire du numéro

Éditeur(s)

Département des relations industrielles de l’Université Laval

ISSN

0034-379X (imprimé)

1703-8138 (numérique)

Découvrir la revue

Citer cet article

(1949). Propos du lecteur : l'avenir de la Loi de la convention collective.

Relations industrielles / Industrial Relations, 4(6), 59-60.

https://doi.org/10.7202/1023494ar

Tous droits réservés (C Département des relations industrielles de l’Université Laval, 1949
Ce document est protégé par la loi sur le droit d'auteur. L’utilisation des services d'Érudit (y compris la reproduction) est assujettie à sa politique d'utilisation que vous pouvez consulter en ligne.

https://apropos.erudit.org/fr/usagers/politique-dutilisation/ 
de la société doit être réformée radicalement. Et elle croit que le syndicalisme ouvrier est un élément de base de cette future société.

Les cadres actuels de la société ne comprennent pas la classe ouvrière. C'est le syndicalisme qui a mission d'intégrer cette classe dans les cadres nouveaux qui s'imposent, puis de lui apprendre les droits et les devoirs qui découleront de cette nouvelle responsabilité.

Il ne s'agit donc pas simplement d'apaiser des revendications ouvrières, encore moins de tirer parti du syndicalisme pour consolider les positions actuelles de la société économique. Il ne s'agit même pas de mettre de l'avant certaines formules qui sans une force pour les contrôler, bien loin d'orienter toute la société vers la poursuite du bien commun, finiraient par transformer les ouvriers eux-mêmes en profiteurs.

Pie XII lui-même, dans son discours aux Associations catholiques des Travailleurs italiens (29 juin 1948) indique «la haute fin vers laquelle doit tendre » le mouvement ouvrier : «la formation des travailleurs vraiment chrétiens qui, excellant également en capacité dans l'exercice de leur art et en conscience religieuse, sachent mettre en harmonie la ferme protection de leurs intérêts économiques avec le sentiment le plus strict de la justice et avec la sincère volonté de collaborer avec les autres classes de la société au renouveau chré. tien de la vie sociale tout entière. »

Voilà tout le programme du Service d'Educa- tion de la C.T.C.C. C'est un véritable souffle de vie qu'il aura le devoir d'entretenir ou de communiquer. Cet organisme répondait à un besoin pressant. Le mouvement syndical catholique et national a tellement besoin de chefs convaincus, fiers de leur organisation professionnelle, éclairés tant au point de vue économique que social, « de témoins vivants d'une doctrine qui ne trompe pas », de chefs qui savent où ils vont et vers quel sommet doit s'orienter le syndicalisme !

Le Service d'Education de la C.T.C.C. se fera un devoir de collaborer avec tous ceux qui s'occupent de formation ouvrière, d'éducation des adultes, il aura besoin de l'expérience de tout le monde pour atteindre sa fin. Il existe lui-même aujourd'hui parce qu'il presse de former plus de syndicalistes militants «qui acceptent que les principes de justice et de charité chrétienne leur coûtent quelque chose », selon l'expression du secrétaire général de la C.T.C.C., M. Jean Marchand.

Le syndicalisme est un élément de réforme, non de révolte, non plus de secourisme de la socité économique actuelle. Il tend à la collaboration avec ceux qui le comprennent. Voilà le problème tel qu'il doit être posé. Il faut être du mouvement, non pour le comprendre, mais pour le sentir, non pour l'expliquer, mais pour le réaliser tout en l'expliquant. - C'est pourquoi la C.T.C.C. a voulu fonder son propre Service d'Education qui posera ce problème et participera à sa solution.

\section{PROPOS DU LECTEUR}

\section{L'avenir de la loi de la convention collective}

En toutes choses, il importe de bien préciser les principes et les faits. Dans le domaine des relations du travail, les interrelations entre ces deux termes sont d'ordinaire spécifiées et concrétisées par des lois: lois générales ou sociologiques, lois particulières ou codifiées; ces lois générales deviennent codifiées lorsqu'elles ont été préalablement confirmées par des habitus ou des faits sociaux.

La situation des relations du travail avant 1934 était plutôt primaire. Aucune législation ou mieux encore une législation précaire venait régir les activités dans ce domaine. Lorsque la loi fut adoptée, il était évident qu'elle avait pour but principal de propager la convention collective à extension juridique. Des faits précis avaient amené l'application d'une telle mesure.

Le législateur s'était rendu compte, d'abord, que la concurrence entre des industries similaires constituait le principal obstacle à la diffusion des conventions collectives de travail. En présence d'une convention collective particulière, l'employeur énonçait l'opinion suivante :
«Vous ne pouvez m'obliger, moi, employeur particulier, à me soumettre à des conditions dont mon principal concurrent est exempté; faites signer cette convention par tous mes concurrents à la fois et en même temps, et j'accepterai moi-même la convention collective que vous me soumettez ainsi, nous serons tous sur un même pied d'égalité ».

Argument d'affaire, étant donné que le salaire est un élément constitutif du prix de revient, l'employeur n'ayant qu'à augmenter proportionnellement son prix de vente pour percevoir un profit équivalent. Mais augmenter obligatoirement un prix de vente lorsqu'un concurrent peut le laisser au mềme niveau, c'est se placer dans une position de concurrence inférieure.

La Loi de la convention collective a pour but de surmonter cet obstacle en décrétant l'extension des clauses d'une convention collective à telle branche de l'activité économique, à condition que les stipulations aient, à la discrétion du ministre, «une signification et une importance prépondérantes $\gg$.

L'intéressant article paru dans l'édition de décembre du Bulletin des relations industrielles de Laval comporte, à mon sens, un éloge trop généralisé de la Loi de la convention collective, non pas que l'éloge n'ait, en soi, sa raison d'être, mais parce que l'auteur semble vouloir faire de la dite loi une panacée universelle qui guérirait tous les maux inhérents aux relations du travail. Il s'agit bien de poser les limites sociologiques d'une telle loi: 
1) La convention collective particulière est à la base de la convention collective à extension juridique.

2) Les clauses d'une convention devant être extensionnées doivent avoir «une signification et une importance prépondérantes $\gg$.

D'abord, il est évident que la convention collective particulière est à la base d'un décret. Ce dernier vient faire l'unité d'ordre, il vient, pour ainsi dire, poser un principe coordinateur de l'activité d'un tout déterminé. Pour ce faire, un tel ajustement demande du temps et de l'étude. Voilà pourquoi, les organisations syndicales, dans leur processus logique, en viennent à l'extension juridique lorsque l'expérience pratique confirme la certitude d'en arriver à l'extension. Une première limite est donc posée dans les faits.

Le législateur a voulu laisser l'autorité de l'application du décret au ministre du Travail. Ici, des prérequis doivent être considérés avant qu'il soit bien défini que les clauses ont «une signification et une importance prépondérantes ». Un de ces prérequis résíde dans le caractère représentatif des agents négociateurs; il importe que les ouviriers de telle industrie soient organisés dans une proportion d'au moins $50 \%$ autrement, il s'agirait bien ici de l'imposition des vues d'une minorité. Du côté de l'employeur autant que du côté de l'employé (puisqu'il incombe à l'employeur de prouver la prépondérance) l'extension juridique occasionne nécessairement des délais inévitables.

D'autres considérations viennent appuyer ces affirmations. La Loi des relations ouvrières, adoptée en 1944, a pour but premier de protéger positivement un droit naturel : le droit d'association. La Loi de la convention collective, bien qu'elle soit chronologiquement antérieure, veut, de par sa nature et sa portée, généraliser les bienfaits apportés par l'exercice du droit d'association pratiquement reconnu en 1944. On voit donc la priorité logique de la Loi des relations ouvrières.

D'autre part, les clauses de salaires d'un décret imposent toujours un minimum afin de conserver le principe «de la signification prépondérante» ce minimum sert souvent de base à la discussion de conventions collectives particulières; tabler sur le décret pour prôner un principe directeur dans les relations patronales-ouvrières, c'est donc minimiser les améliorations apportées par les législations concurrentes.

Disons enfin, que la mise en vigueur des décrets a donné naissance à certains abus qui proviennent, non pas de la carence du principe même de la loi, mais de la difficulté d'application d'un tel principe. En présence du décret-loi, certains employeurs ont subi un réflexe défensif assez intéressant. Ils ont tenté de se servir du décret pour établir des monopoles en faisant jouer leur influence pour hausser le plus possible les taux de salaires (ce qui peut paraître assez étrange, à première vue).

Il arrive nécessairement que dans une industrie donnée, les entreprises n'ont pas toute la même importance économique. Les grosses entreprises s'efforçaient donc de hausser le minimum le plus possible afin d'engloutir les très petites entreprises en les plaçant dans l'impossibilité économique de payer le salaire décrété. Le zonage n'a pas apporté un plein remède à cet état de chose, non pas qu'il remette en cause l'obstacle premier à savoir : des échelles de salaire différenciées, mais parce que l'activité économique actuelle comporte des entreprises réparties d'une face fort disproportionnée dans une industrie donnée. La situation serait équilibrée, si l'on avait en présence des industries moyennes; mais, la juxtaposition de très grosses et de très petites entreprises vient compliquer l'application pratique du décret alors que l'application heureuse d'une loi présuppose une activité économique normale c'est-à-dire équilibrée.

Il est bien d'encourager et de préconiser la multiplication des conventions collectives à extension juridique, mais il importe d'en examiner les possibilités forcément limitées. Lorsqu'on l'étudie sous cet angle, on ne veut pas \& faire valoir les raisons de limiter le champ d'action de la loi » comme dit l'auteur de l'article, mais on veut tout simplement placer la loi dans le champ d'action qui lui est propre.

Enfin, il me semble que c'est une opinion erronée de croire que l'on peut arriver à «étudier, chercher et trouver le moyen de l'étendre (la loi de la convention collective) à tout le domaine et à tous les problèmes des relations patronales-ouvrières ». Le législateur s'attarde présentement à l'étude d'un Code du Travail, il n'est pas téméraire d'énoncer qu'un tel code ne peut comporter uniquement la convention collective améliorée pouvant être étendue à « tout le domaine et à tous les problèmes des relations patronales-ouvrières $\gg$.

Il ne peut $y$ avoir un unique remède aux maladies du corps social d'aujourd'hui: la synthèse, la plénitude d'une législation doit couvrir tous les échelons et doit protéger tous les droits.

\section{La direction du personnel de commerce de détail}

Le Département des relations industrielles organise conjointement avec le Service extérieur d'éducation sociale de l'Université Laval, la tenue d'une session intensive qui débutera le 14 mars prochain à deux heures de l'aprèsmidi, pour se terminer le 15 , à midi. Cette session portera sur la direction du personnel dans le commerce de détail. Le programme comprend quatre séances, comme suit:

\section{LUNDI, 14 MARS:}

Introduction da la direction du personnel en général: de 2.00 à 3.30 p.m., Me Jean Gagné, B.A., LL.L., M.Sc.Sco., avocat, professeur et secrétaire du Département des relations industrielles de l'Université Laval.

Le probleme de la direction du personnel dans un magasin de détail: Justification, organisation et fonctionne- ment d'un tel service: de 3.30 à 5.00 p.m., M. JeanMathieu Cantin, M.Sc.Soc., Directeur du personnel, Le Syndicat de Québec Ltée.

Le rôle et la fonction d'un chef de rayon dans un magasin de détail: de 8.00 à 10.30 p.m., M. Edouard Coulombe, B.A., gérant général, J.-B. Laliberté Ltée, président, Conseil central des associations patronales de Québec.

MARDI, 15 MARS:

L'administration de la convention collective et la formation du personnel dans le commerce de détail: de 9.30 à 12.00 a.m., M. Louis Bilodeau, secrétaire, Association patronale des services hospitaliers de l'est de la province de Québec.

Les frais d'inscription à ces cours sont de $\$ 10.00$ (souper du lundi inclus). La session se tiendra à l'Institut Canadien, 37, rue Ste-Angèle, Québec. On est prié de s'inscrire au plus tard le 8 mars prochain, au Service extérieur d'éducation sociale, 2 rue de l'Université, Québec. 